Louisiana State University

LSU Digital Commons

Faculty Publications

Department of Physics \& Astronomy

7-1-2005

\title{
Attosecond electron wave packet dynamics in strong laser fields
}

P. Johnsson

Lunds Tekniska Högskola

R. López-Martens

Laboratoire d'Optique Appliquée

S. Kazamias

Universite Paris-Saclay

J. Mauritsson

Louisiana State University

C. Valentin

Laboratoire d'Optique Appliquée

See next page for additional authors

Follow this and additional works at: https://digitalcommons.Isu.edu/physics_astronomy_pubs

\section{Recommended Citation}

Johnsson, P., López-Martens, R., Kazamias, S., Mauritsson, J., Valentin, C., Remetter, T., Varjú, K., Gaarde, M., Mairesse, Y., Wabnitz, H., Salières, P., Balcou, P., Schafer, K., \& L'Huillier, A. (2005). Attosecond electron wave packet dynamics in strong laser fields. Physical Review Letters, 95 (1) https://doi.org/10.1103/ PhysRevLett.95.013001

This Article is brought to you for free and open access by the Department of Physics \& Astronomy at LSU Digital Commons. It has been accepted for inclusion in Faculty Publications by an authorized administrator of LSU Digital Commons. For more information, please contact ir@lsu.edu. 


\section{Authors}

P. Johnsson, R. López-Martens, S. Kazamias, J. Mauritsson, C. Valentin, T. Remetter, K. Varjú, M. B. Gaarde, Y. Mairesse, H. Wabnitz, P. Salières, Ph Balcou, K. J. Schafer, and A. L'Huillier 


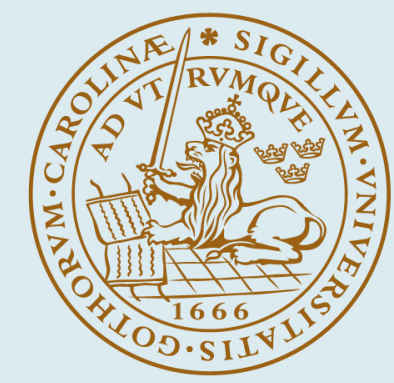

\section{LUND UNIVERSITY}

\section{Attosecond electron wave packet dynamics in strong laser fields}

Johnsson, Per; Lopez-Martens, R; Kazamias, S; Mauritsson, J; Valentin, C; Remetter, Thomas; Varju, Katalin; Gaarde, M B; Mairesse, Y; Wabnitz, H; Salieres, P; Balcou, P; Schafer, K J; L'Huillier, Anne

Published in:

Physical Review Letters

DOI:

10.1103/PhysRevLett.95.013001

2005

Link to publication

Citation for published version (APA):

Johnsson, P., Lopez-Martens, R., Kazamias, S., Mauritsson, J., Valentin, C., Remetter, T., Varju, K., Gaarde, M. B., Mairesse, Y., Wabnitz, H., Salieres, P., Balcou, P., Schafer, K. J., \& L'Huillier, A. (2005). Attosecond electron wave packet dynamics in strong laser fields. Physical Review Letters, 95(1), 013001-1-013001-4. https://doi.org/10.1103/PhysRevLett.95.013001

Total number of authors:

14

\footnotetext{
General rights

Unless other specific re-use rights are stated the following general rights apply:

Copyright and moral rights for the publications made accessible in the public portal are retained by the authors and/or other copyright owners and it is a condition of accessing publications that users recognise and abide by the legal requirements associated with these rights.

- Users may download and print one copy of any publication from the public portal for the purpose of private study or research.

- You may not further distribute the material or use it for any profit-making activity or commercial gain

- You may freely distribute the URL identifying the publication in the public portal
}

Read more about Creative commons licenses: https://creativecommons.org/licenses/

Take down policy

If you believe that this document breaches copyright please contact us providing details, and we will remove access to the work immediately and investigate your claim. 


\title{
Attosecond Electron Wave Packet Dynamics in Strong Laser Fields
}

\author{
P. Johnsson, ${ }^{1}$ R. López-Martens, ${ }^{2}$ S. Kazamias, ${ }^{3}$ J. Mauritsson, ${ }^{4}$ C. Valentin, ${ }^{2}$ T. Remetter, ${ }^{1}$ K. Varjú,,${ }^{1, *}$ M. B. Gaarde, ${ }^{4}$ \\ Y. Mairesse, ${ }^{5}$ H. Wabnitz, ${ }^{5}$ P. Salières, ${ }^{5} \mathrm{Ph}$. Balcou, ${ }^{2}$ K. J. Schafer, ${ }^{4}$ and A. L'Huillier ${ }^{1}$ \\ ${ }^{1}$ Department of Physics, Lund Institute of Technology, P.O. Box 118, SE-221 00 Lund, Sweden \\ ${ }^{2}$ Laboratoire d'Optique Appliquée, Ecole Nationale Supérieure des Techniques Avancées (ENSTA) - Ecole Polytechnique \\ CNRS UMR 7639, 91761 Palaiseau Cedex, France \\ ${ }^{3}$ Laboratoire d'Interaction du rayonnement X Avec la Matière, Université Paris-Sud, 91405 Orsay, France \\ ${ }^{4}$ Department of Physics and Astronomy, Louisiana State University, Baton Rouge, Louisiana 70803-4001, USA \\ ${ }^{5}$ CEA/DSM/DRECAM/SPAM, Bât. 522, Centre d'Etudes de Saclay, 91191 Gif-sur-Yvette, France
}

(Received 14 December 2004; published 29 June 2005)

\begin{abstract}
We use a train of sub-200 attosecond extreme ultraviolet (XUV) pulses with energies just above the ionization threshold in argon to create a train of temporally localized electron wave packets. We study the energy transfer from a strong infrared (IR) laser field to the ionized electrons as a function of the delay between the XUV and IR fields. When the wave packets are born at the zero crossings of the IR field, a significant amount of energy $(\sim 20 \mathrm{eV})$ is transferred from the field to the electrons. This results in dramatically enhanced above-threshold ionization in conditions where the IR field alone does not induce any significant ionization. Because both the energy and duration of the wave packets can be varied independently of the IR laser, they are valuable tools for studying and controlling strong-field processes.
\end{abstract}

DOI: 10.1103/PhysRevLett.95.013001

Attosecond pulses constitute a novel tool for probing processes taking place on the time scale of electron motion inside atoms. They have been produced in the extreme ultraviolet (XUV) region both as attosecond pulse trains (APTs) [1-5] and as single attosecond pulses [6-10] via high harmonic generation [11]. APTs, synthesized from several phase-locked harmonics, promise to be particularly flexible attosecond sources since the amplitude and relative phase of the spectral components (the harmonics) can be tailored to vary the duration and the time-frequency characteristics of the pulses [5,12]. Exploiting this flexibility offers a new route to controlling strong-field interactions in atoms and molecules.

Strong-field processes are initiated by the creation of temporally confined electron wave packets (EWPs) which subsequently gain energy from a strong infrared (IR) field. The interaction of these wave packets with their parent ion gives rise to processes such as nonsequential double ionization, high-order harmonic generation, and abovethreshold ionization (ATI) [11,13-15], and has recently been exploited in molecular clock experiments [16-19]. In the most common scenario, the same intense IR field also drives the EWP creation via tunneling through the suppressed Coulomb barrier. These tunneling EWPs are formed periodically near the peak of the electric field cycle, and their properties are determined almost solely by the IR intensity. EWPs produced by APTs, on the other hand, directly inherit their properties through the singlephoton ionization step. Thus, the energy, timing, and coherence of the EWPs can be varied independently of the process under investigation by controlling the properties of the APT. This opens up a new range of experiments, where APTs can be used as a controllable injection mechanism for EWPs in strong-field experiments [20].
PACS numbers: $32.80 . \mathrm{Rm}, 32.80 . \mathrm{Qk}, 42.65 . \mathrm{Ky}$

In this Letter, we use well characterized APTs, with central energy $\hbar \omega_{\mathrm{XUV}} \approx 30 \mathrm{eV}$, to generate EWPs near the ionization threshold in argon, and we study the subsequent dynamics of these wave packets in a strong IR field. The major obstacle to performing this type of experiment was recently overcome with the generation of true ontarget APTs [5]. We show that the exchange of energy between the EWP and an intense optical field, a crucial step in all strong-field processes, is greatly affected both by changing the timing of the attosecond pulses and by altering their coherence properties (frequency chirp). Since the EWPs are injected close to the ionization threshold, the presence of the atomic potential also manifests itself during the energy exchange with the IR field. This is in contrast with previous attosecond experiments using EWPs with high energies where effects due to the ion core are negligible $[7,8]$. The use of APTs to control both the timing of the EWP creation and its subsequent energy exchange with the IR field represents the first application of APTs.

When a temporally confined EWP is injected into the continuum in the presence of an IR field, the generated photoelectrons will be redistributed in energy depending on the IR intensity and the initial properties of the EWP, as well as on the timing of the injection with respect to the IR cycle. For an EWP injected with a high initial momentum, the presence of the Coulomb potential can be neglected and the cross section for absorption or emission of IR photons can be assumed to be constant over the energy range. In this case, the final momentum of an electron injected at time $t_{i}$, with an initial momentum in the direction of the IR field polarization, will be simply given by $p_{f}\left(t_{i}\right)=p\left(t_{i}\right)-$ $e A\left(t_{i}\right)$, where $p\left(t_{i}\right)$ is the initial momentum of the EWP, $e$ is the electron charge, and $A\left(t_{i}\right)$ the vector potential of the IR 
field at the time of injection [21]. If an APT synthesized from high-order harmonics is used for the injection, EWPs are generated periodically with a separation of half a laser cycle, leading to the appearance of peaks spaced by $2 \hbar \omega$ in the photoelectron spectrum. In the presence of an IR field, two consecutive EWPs will be shifted in momentum in opposite directions since $A\left(t_{i}\right)=-A\left(t_{i}+\frac{\pi}{\omega}\right)$, giving two contributions, with different energy, to the final photoelectron distribution. In the time domain, the periodicity of the process becomes equal to the full laser cycle, and peaks spaced by $\hbar \omega$ appear in the photoelectron spectrum. At low IR intensities these are seen as sidebands in between the harmonic peaks, the intensities of which vary with delay [1]. For high IR intensities, electrons with energies well outside the original one-photon electron spectrum will be produced at certain delays. In our case, the APTs are synthesized from plateau harmonics and the EWPs are injected with low initial momentum, so that the effect of the Coulomb potential cannot be neglected. This will lead to an asymmetry between the low and high energy parts of the spectrum.

In order to illustrate the effect of a strong IR field and that of the Coulomb potential we have calculated the photoelectron spectra by integrating the time-dependent Schrödinger equation (TDSE) in three dimensions for an argon atom in the presence of a strong $800 \mathrm{~nm}$ IR field and an APT [22], including electrons emitted within a $2 \pi$ solid angle. The IR intensity $\left(3 \times 10^{13} \mathrm{~W} \cdot \mathrm{cm}^{-2}\right)$ and the time structure of the APT are the same as in the experiment. At these IR intensities the ionization is dominated by the APT. The results are presented in Figs. 1(a) and 1(b), together with illustrations of the relationship between the fields, and in Fig. 1(c) the total photoelectron spectrum is shown as a function of the delay $\tau$ between the two fields. Throughout this Letter, the phase delay $\omega \tau$ is given relative to a sine wave. When the pulses in the APT coincide with the peaks of the IR field $[\omega \tau=(n+1 / 2) \pi$, Fig. 1(a)], the vector potential is zero and so the average momentum change of the wave packet is as well. When the pulses instead coincide with the zero crossings of the IR field [ $\omega \tau=n \pi$, Fig. 1(b)], the magnitude of the vector potential, and thus the momentum change, is maximum [7,8,21], and the electron energy distribution is much broader. The reason why we see a broadening, and not just a shift, is that the contributions from two consecutive EWPs are shifted in opposite directions in energy as discussed above. However, the probability of emitting IR photons is significantly decreased, compared to the probability of absorbing IR photons, when the final state approaches the ionization threshold [23]. This leads to a "pileup" of electrons at low energies, whereas the high energy side can expand freely.

Because of the finite duration of the EWP, different parts of the wave packet are born at different instants of the IR field, and thus experience a different momentum change. If the injected EWP is transform limited (i.e., the central
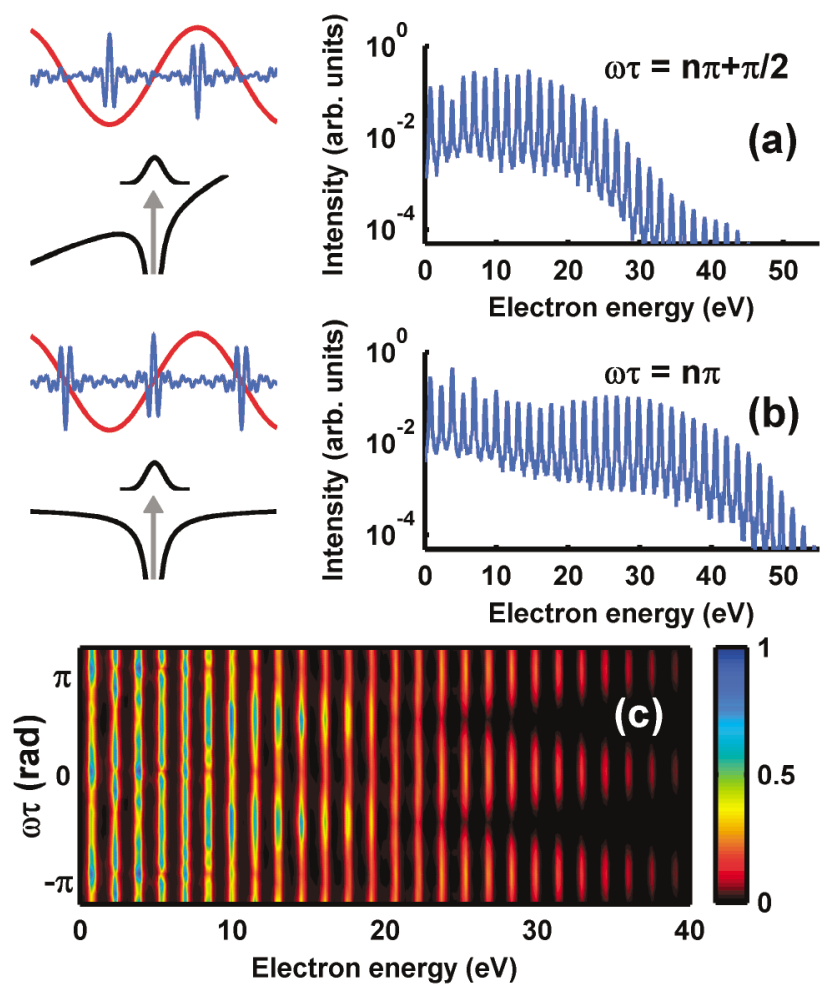

FIG. 1 (color). Controlling ATI using an attosecond pulse train. On the left side the timing between the two fields, together with the shape of the atomic potential at the moment when the wave packet is released into the continuum, are depicted. On the right side photoelectron spectra calculated by solving the TDSE for the two cases (a) and (b) are presented on a logarithmic scale. In (c) the calculated photoelectron spectrum as a function of the delay is shown on a linear scale.

frequency of the APT is constant), $p\left(t_{i}\right)$ is constant over the pulse duration, leading to a broadening of the photoelectron spectral envelope for all delays. If instead the EWP is initially chirped (i.e., has an energy variation over its temporal duration), $p\left(t_{i}\right)$ will vary over the pulse duration and the energy distribution will be either further broadened, if the derivatives of $p\left(t_{i}\right)$ and $A\left(t_{i}\right)$ have the same sign, or narrowed, if the sign is opposite $[8,24]$. The influence on the electron energy distribution will be further discussed in the experimental section.

Our experimental method for generating, characterizing, and manipulating APTs has been described in detail elsewhere $[5,25]$. Briefly, the APT is synthesized from harmonics 13 through 35 generated in argon from an $800 \mathrm{~nm}$, 35 fs Ti:sapphire laser pulse. The harmonics are filtered using a hard aperture and aluminum foils. The aperture is used to remove the contribution from the long quantum path to the harmonic emission, while the aluminum filters remove the remaining IR and the intense low-order harmonics. These filters also serve to compress the attosecond pulses using the negative group-delay dispersion of aluminum to compensate for the intrinsic positive chirp of the attosecond pulses [2]. The APT is overlapped both spa- 
tially and temporally with the dressing field (a delayed replica of the IR pulse used for harmonic generation). The two beams copropagate collinearly and are then focused with the same focusing mirror into a detection gas (argon) inside a magnetic bottle electron time-of-flight spectrometer (MBES). The MBES collects electrons emitted within a $2 \pi$ solid angle around a direction parallel to the polarization of the IR and XUV fields. The static pressure of the detection gas is $\sim 1 \times 10^{-4}$ mbar and care is taken to avoid space-charge effects during the experiment. By applying different retarding potentials to the flight tube of the MBES, we resolve different energy regions in the photoelectron spectrum. We can characterize the EWP at the time of injection by using a weak IR intensity, such that only first order sidebands are produced. The spectral interference in these sidebands, along with the photoelectron peak amplitudes, can be used to reconstruct the average time structure of the EWP at the time of injection $[1,26]$. This technique is often referred to as RABITT (reconstruction of attosecond beating by interference of two-photon transitions) [26]. In addition, the maxima of the sideband oscillations appear when the EWPs are created at the peaks of the IR field, allowing us to unambiguously determine the absolute delay between the EWP and the dressing IR field. Furthermore, by correcting for the single-photon ionization cross section, the harmonic amplitudes can be determined from the measured photoelectron peak amplitudes. Finally, by repeating the measurement for different detection gases so that all harmonics on target are included, the average shape of the on-target attosecond XUV pulses can be reconstructed.

The current experiment was performed using two attosecond pulse durations. Using a $600 \mathrm{~nm}$ thick aluminum filter (Fig. 2), the intrinsic positive chirp of the attosecond pulses is almost completely compensated for [5], yielding an average of 160 as per pulse in the APT and 180 as per wave packet in the EWP train at the time of their injection. The EWPs are longer than the XUV pulses due to the reduction in bandwidth imposed by the ionization cross section of argon. The effect of a frequency modulation on the EWPs was investigated by using a $200 \mathrm{~nm}$ aluminum filter (Fig. 3). With this filter thickness, both the attosecond XUV pulses and the EWPs exhibit a positive chirp. Here, the average duration of each pulse in the APT is 340 as, whereas that of the EWPs is shortened to 260 as due to spectral filtering caused by the ionization process.

Figure 2(a) presents experimental photoelectron spectra for different conditions: only the IR field (blue line), only the APT (black line), both beams with $\omega \tau=n \pi$ (red line), and $\omega \tau=\left(n+\frac{1}{2}\right) \pi$ (green line). The IR intensity $\left(I_{\mathrm{IR}} \approx\right.$ $\left.3 \times 10^{13} \mathrm{~W} \cdot \mathrm{cm}^{-2}\right)$ is not strong enough to induce any significant ATI (a few low-order peaks are visible) and the ionization is mainly dominated by the APT when present. The short duration of the attosecond pulses allows us to control the instant of ionization relative to the IR field and,

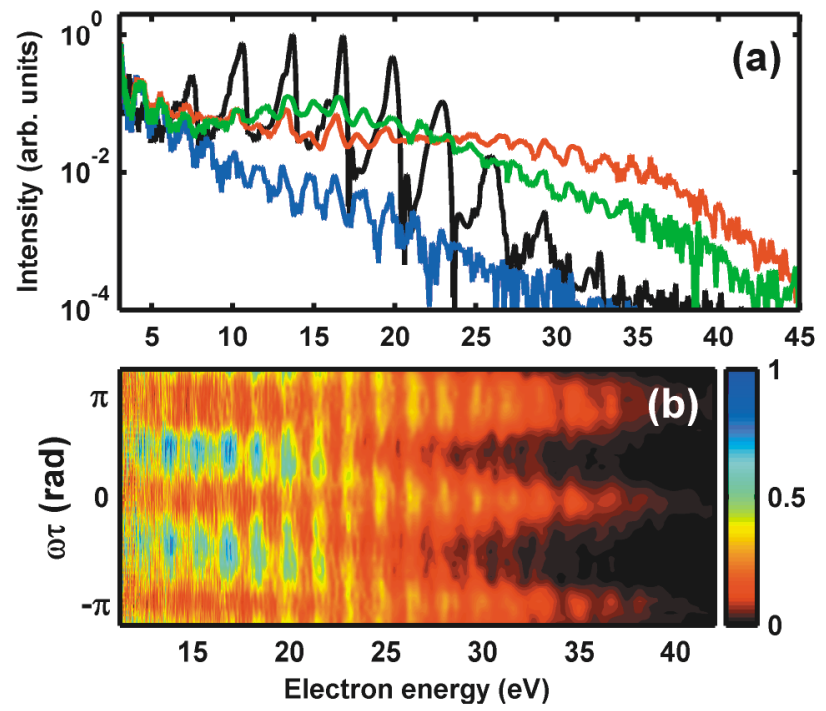

FIG. 2 (color). Experimental results. (a) Photoelectron spectra (logarithmic scale) obtained by concatenation of measurements for different retarding potentials, in different conditions: with the IR field alone at an intensity equal to $3 \times 10^{13} \mathrm{~W} \cdot \mathrm{cm}^{-2}$ (blue line), with a train of 160 as pulses alone (black line), with both fields overlapped at $\omega \tau=n \pi$ (red line), and $\omega \tau=\left(n+\frac{1}{2}\right) \pi$ (green line). (b) Photoelectron spectra as a function of time delay between the XUV pulses and the IR field (linear scale), taken with a retarding potential of $9.3 \mathrm{~V}$.

in particular, to choose time intervals not accessible through tunneling ionization (i.e., $\omega \tau=n \pi$ ). A detailed experimental study is presented in Fig. 2(b), which shows how the photoelectron signal varies as a function of energy and time delay over slightly more than one cycle of the IR light ( 1 cycle corresponds to $2.6 \mathrm{fs}$ ). The periodic displacement of the energy distribution towards higher energies as $\tau$ is varied is quite noticeable, directly reflecting the effect of creating a temporally localized EWP at the zero crossings of an intense IR field.

In a semiclassical model [9,21], which neglects the ionization potential, the maximum final energy of an electron in an IR dressing field is given by $W_{f}=W_{\mathrm{EWP}}+$ $2 U_{p}+\sqrt{8 W_{\mathrm{EWP}} U_{p}}$, where $W_{\mathrm{EWP}}$ is the initial energy of the electron and $U_{p}$ is the ponderomotive energy. In our

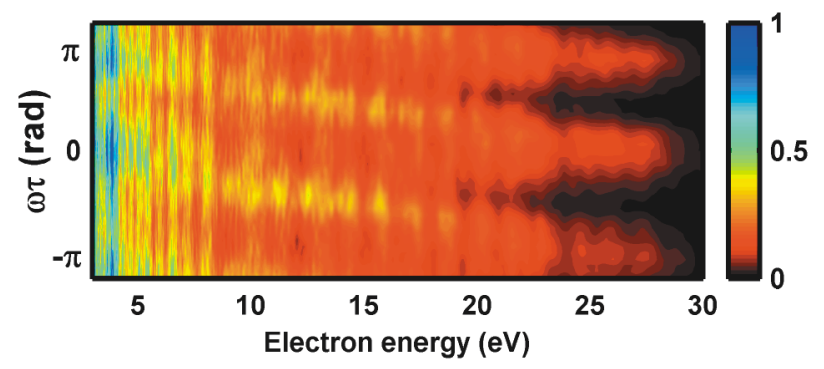

FIG. 3 (color). Photoelectron spectra as a function of time delay between the XUV pulses and the IR field, recorded with chirped 340 as pulses and no retarding potential. 
case, $W_{\mathrm{EWP}} \approx 15 \mathrm{eV}$ and $U_{p} \approx 2 \mathrm{eV}$ such that $W_{f} \approx$ $35 \mathrm{eV}$. Comparing this with the cutoff law for ATI $\left(10 U_{p} \approx 20 \mathrm{eV}\right)[27,28]$, we see that using an APT as an injection mechanism for EWPs makes it possible to produce electrons at energies not normally accessible with the IR field alone. The transfer of energy to the electron is somewhat affected by the atomic potential, as exemplified by the asymmetry between the low and the high energy parts of the spectrum. Although the experiment includes averaging effects, due to pulse-to-pulse variations in the APT [29], the finite length of the dressing field, and volume effects, the agreement between our experimental data (Fig. 2) and the theoretical single-atom predictions (Fig. 1), which were calculated using the experimental parameters, is striking.

We have also investigated the influence of a frequency modulation (chirp) of the attosecond pulses and consequently of the EWPs on their interaction with the IR field. Figure 3 shows a result obtained for chirped (340 as) pulses. When the momentum change of the EWPs goes through zero $\left(\omega \tau= \pm \frac{\pi}{2}\right)$, the contributions to the electron spectrum due to two consecutive attosecond pulses do not have the same spectral width. The narrower contribution will dominate the resulting photoelectron spectrum, leading to an asymmetric electron distribution relative to zero delay [in contrast to Fig. 2(b)]. The observation of these effects clearly indicates the possibility of controlling continuum dynamics not only by controlling the timing, but also the time-frequency characteristics of the EWPs.

In conclusion, we have generated attosecond EWPs with a duration of less than 200 as close to threshold in argon using tailored APTs. Furthermore, we studied their interaction with a strong IR field. With the pulse parameters used, the ionization is dominated by the APT and tunable ATI plateaus appear as an effect of the dressing field. The strong delay dependence seen for these ATI spectra is a direct consequence of the confinement of the EWPs to a shorter time than the period of the IR field. These results open the door to the generation of tunable and well characterized attosecond EWPs which are essential for the study and control of a large number of atomic and molecular strong-field processes, such as harmonic generation and multiple ionization [20,30].

This research was supported by the Marie Curie European Programme (MEIF-CT-2004-009268; MRTNCT-2003-505138, XTRA), the Access to Research Infrastructures activity in the Sixth Framework Programme of the EU (Contract No. RII3-CT-2003506350, Laserlab Europe), the Knut and Alice Wallenberg Foundation, the Swedish Science Council, and the National Science Foundation through Grant No. PHY-0401625. M. G. acknowledges the support of the Louisiana Board of Regents through Grant No. LEQSF(2004-07)-RD-A-09.

*On leave from Department of Optics and Quantum Electronics, University of Szeged, Szeged, Hungary.

[1] P. M. Paul et al., Science 292, 1689 (2001).

[2] Y. Mairesse et al., Science 302, 1540 (2003).

[3] S. A. Aseyev, Y. Ni, L. J. Frasinski, H. G. Muller, and M. J. J. Vrakking, Phys. Rev. Lett. 91, 223902 (2003).

[4] P. Tzallas et al., Nature (London) 426, 267 (2003).

[5] R. López-Martens et al., Phys. Rev. Lett. 94, 033001 (2005).

[6] M. Hentschel et al., Nature (London) 414, 509 (2001).

[7] R. Kienberger et al., Science 297, 1144 (2002).

[8] R. Kienberger et al., Nature (London) 427, 817 (2004).

[9] M. Drescher et al., Nature (London) 419, 803 (2002).

[10] E. Goulielmakis et al., Science 305, 1267 (2004).

[11] M. Ferray et al., J. Phys. B 21, L31 (1988).

[12] K. T. Kim, C. M. Kim, M. G. Baik, G. Umesh, and C. H. Nam, Phys. Rev. A 69, 051805 (2004).

[13] P. Agostini, F. Fabre, G. Mainfray, G. Petite, and N. K. Rahman, Phys. Rev. Lett. 42, 1127 (1979).

[14] K. J. Schafer, B. Yang, L. F. DiMauro, and K. C. Kulander, Phys. Rev. Lett. 70, 1599 (1993).

[15] P. B. Corkum, Phys. Rev. Lett. 71, 1994 (1993).

[16] H. Niikura et al., Nature (London) 417, 917 (2002).

[17] H. Niikura et al., Nature (London) 421, 826 (2003).

[18] A. S. Alnaser et al., Phys. Rev. Lett. 93, 183202 (2004).

[19] F. Légaré et al., Phys. Rev. Lett. 91, 093002 (2003).

[20] K. J. Schafer, M. B. Gaarde, A. Heinrich, J. Biegert, and U. Keller, Phys. Rev. Lett. 92, 023003 (2004).

[21] F. Quéré, Y. Mairesse, and I. Itatani, J. Mod. Opt. 52, 339 (2005).

[22] K. C. Kulander, K. J. Schafer, and J. L. Krause, in Atoms in Intense Laser Fields, edited by M. Gavrila (Academic, New York, 1992).

[23] R. Taïeb et al., J. Opt. Soc. Am. B 13, 363 (1996).

[24] J. Itatani et al., Phys. Rev. Lett. 88, 173903 (2002).

[25] R. López-Martens et al., Appl. Phys. B 78, 835 (2004).

[26] H. G. Muller, Appl. Phys. B 74, S17 (2002).

[27] M. Lewenstein, K. C. Kulander, K. J. Schafer, and P. H. Bucksbaum, Phys. Rev. A 51, 1495 (1995).

[28] W. Becker, A. Lohr, and M. Kleber, J. Phys. B 27, L325 (1994).

[29] K. Varjú et al., J. Mod. Opt. 52, 379 (2005).

[30] P. Antoine et al., in Proceedings of the 7th International Conference on Multiphoton Processes, Institute of Physics Conference Series Number 154, edited by P. Lambropoulos and $\mathrm{H}$. Walther (Institute of Physics Publishing, Berkshire, 1996), p. 142. 